\title{
Data Analysis Services Related to the IoT and Big Data: Potential Business Opportunities for Third Parties
}

\author{
Izabella V. Lokshina \\ SUNY Oneonta \\ Izabella.Lokshina@oneonta.edu
}

\author{
Barbara J. Durkin \\ SUNY Oneonta \\ Barbara.Durkin@oneonta.edu
}

\author{
Cees J. M. Lanting \\ DATSA Belgium, Consulting \\ Cees.Lanting@gmail.com
}

\begin{abstract}
The Internet of Things (IoT) provides the tools for the development of a major, global data-driven ecosystem. When accessible to people and businesses, this information can make every area of life, including business, more data-driven. In this ecosystem, with its emphasis on Big Data, there has been a focus on building business models for the provision of services, the so-called Internet of Services (IoS). These models assume the existence and development of the necessary IoT measurement and control instruments, communications infrastructure, and easy access to the data collected and information generated by any party. Different business models may support opportunities that generate revenue and value for various types of customers. This paper contributes to the literature by considering business models and opportunities for third-party data analysis services and discusses access to information generated by third parties in relation to Big Data techniques and potential opportunities.
\end{abstract}

\section{Introduction}

The much discussed Internet of Things (IoT) provides a set of tools enabling a major, global datadriven ecosystem to develop devices (or Things) encompassing everything from pedometers to seismographs, collect data and produce unprecedented amounts of information about the parameters and items in the world around us. When put in the hands of people and businesses, this information can make every area of life, including business, more datadriven.

Things are not really a new concept. We've been using sensors to collect scientific data for centuries. What's different now is the interconnection of all these devices, producing ever more granular data sets, all while that data is becoming more and more accessible, potentially to everyone. To actually put that data to work, we need to make sense of it. When massive amounts of data become accessible and understandable, the implications are enormous for civic life, personal health and business.

Until now, much attention and effort have gone in the development of business models, in particular for the provision of services in this data-driven ecosystem in the context of IoT, sometimes referred to as the Internet of Services (IoS). These business models assume the existence and development of the necessary IoT measurement and control instruments, communications infrastructure, and easy access to the data collected and information generated by any party. However, not every business model may support opportunities that generate revenue or value or are suitable for different types of customers. Other business models should also be considered.

A discussion of the infrastructure is outside the scope of this paper. For our purposes, we will assume that a significant time will be needed for deployment. Regulatory clarity and permission must be obtained. Privacy and possible national security issues must be addressed.

This paper contributes to the literature by reviewing, for the first time, business models and opportunities for third party data analysis services. It discusses access to information generated by third parties as a prerequisite to data analysis services and, in particular, in relation to Big Data techniques and potential opportunities. Strategic implications for small businesses that use the IoT in data-driven ecosystems are considered.

This paper is comprised of eight sections and is organized as follows. Section two provides an overview of the generation of data and of value and discusses data access and ownership issues. Section three considers building business models in global data-driven ecosystems. Section four focuses on dataand IoT service-based business models. Section five provides an analysis of Big Data techniques and describes strategic implications and business opportunities. Section six gives an overview of experiences of European small and medium enterprises related to IoT and Big Data. Section seven contains the summary and conclusions. Finally, section eight lists references. 


\section{Generation of data and value}

The example of a commercial airliner provides a scenario for understanding how IoT data is collected and information is generated. For the purposes of controlling the airplane and verifying the correct operation of its components, a multitude of parameters are measured. The values of these parameters are recorded (and, in real-time, analyzed for artifacts). Additional parameters are recorded for off-line analysis, for maintenance purposes or for use in case of an incident (e.g., a "black box"). Additional data is collected (and possibly recorded) outside the plane, such as the flight path (e.g., by air traffic control), weather (e.g., by meteorological services), and information from nearby planes. Further information may be available from sources that are not professionally or institutionally related, such as video and photographic material generated by aviation enthusiasts. The data corresponding to operation and the components that are specifically recorded in the plane are owned by the specific parties according to agreements among the manufacturer, supplier, owner, and user, in compliance with the rules established by the aeronautics authorities. The additional data collected outside the plane is likely under different ownership subject to the control of professional or institutional-related organizations. However, access and ownership to the data from other sources, such as aviation enthusiasts, may be subject to other rules.

The concept of the Internet of Things is not really new. It is, however, changing the game for data access. When people can actually visualize and interact with this data and information, even blend it with their organization's other data assets and knowledge, entirely new insights can be reached. From jet engines bolted on airliners to pacemakers embedded in hearts, the increasingly interconnection of devices around us means that we can see our world in completely new ways. Ultimately, this empowers innovation that's not only data-driven but could also be human-centric.

Big Data is defined as high-volume, high-velocity and/or high-variety information assets that demand cost-effective, innovative forms of information processing that enable enhanced insight, decision making, and process automation [3]. Big Data does not arise out of a vacuum; it is recorded from some data-generating source. For example, consider our ability to sense and observe the world around us, from the heart rate of an elderly citizen and the presence of toxins in the air we breathe to the planned square kilometer array telescope (which will produce up to 1 million terabytes of raw data per day). Similarly, scientific experiments and simulations can easily produce petabytes of data today [3, 22, 23].
Much of this data, when considered in isolation, appears to be of no interest and can be filtered and compressed by orders of magnitude. One challenge is to define these filters in such a way that they do not discard useful information. We need research in the science of data reduction that can intelligently process this raw data to a size that its users can handle while not missing the needle in the haystack. Furthermore, we require online analysis techniques that can process such streaming data on the fly since we cannot afford to store first and reduce afterward.

The second big challenge is to automatically generate the right metadata to describe what data is recorded as well as how it is recorded and measured. Another important issue here is data provenance (i.e., the source of data). Recording information about the data at its birth is not useful unless this information can be interpreted and carried along through the data analysis process.

Generation of data and value in the context of Big Data involves multiple distinct phases, each of which introduces challenges. While the promise of Big Data is real, there is currently a big gap between its potential and its realization. Heterogeneity, scale, timeliness, complexity, and privacy problems with Big Data impede progress at all phases of the process that can create value from data. Data analysis, organization, retrieval, and modeling are other foundational challenges.

Data analysis is a process of inspecting, cleaning, transforming, and modeling data with the goal of discovering useful information, suggesting conclusions, and supporting decision-making. Data analysis has multiple facets and approaches, encompassing diverse techniques in business, science, and social science domains. This includes data mining, business intelligence and statistical applications such as descriptive statistics, exploratory data analysis, and confirmatory data analysis; predictive analytics that focus on the application of statistical models for predictive forecasting or classification; and text analytics that apply statistical, linguistic, and structural techniques to extract and classify information from textual sources. Data integration is a precursor to data analysis. Data analysis is closely linked to data visualization and data dissemination.

Data analysis is a clear bottleneck in many applications, both due to the lack of scalability of the underlying algorithms and the complexity of the data that needs to be analyzed. Finally, presentation of the results and its interpretation by non-technical domain experts is crucial to extracting actionable knowledge.

For more than three decades, data management principles such as physical and logical independence, declarative querying and cost-based optimization have 
led to a multi-billion-dollar industry. More importantly, these technical advances have enabled the first round of business intelligence applications and laid the foundation for managing and analyzing Big Data today.

The many novel challenges and opportunities associated with Big Data necessitate rethinking many aspects of these data management platforms while retaining other desirable aspects. Appropriate investment in Big Data will lead to a new wave of fundamental technological advances that will be embodied in the next generations of Big Data management and analysis platforms, products, and systems. These research problems are not only timely but also having the potential to create significant economic value in the American economy for years to come. However, these problems are also difficult, requiring us to rethink data analysis systems in fundamental ways. A major investment in Big Data, properly directed, can result not only in major scientific advances, but also in laying the foundation for the next generation of advances in science, medicine, and business.

The privacy of data is one of several significant concerns that increase in the context of Big Data. For electronic health records, there are strict laws governing what can and cannot be done. For other data, regulations are less forceful, particularly in the United States. However, there is a great deal of public fear regarding the inappropriate use of personal or corporate data, particularly through linking of data from multiple sources. Managing privacy is effectively a technical, sociological and legal problem which must be addressed jointly from all perspectives to realize the promise of Big Data. Consider, for example, data gleaned from location-based services. These new architectures would require users to share their location with the service provider, resulting in obvious privacy concerns. Note that hiding the identity of users alone without hiding their locations would not properly address these privacy concerns. An attacker or a potentially malicious location-based server can infer the identity of the query source from its subsequent location information. For example, a user's location information can be tracked through several stationary connection points (e.g., cell towers). After a while, the user leaves a trail which could be associated to a certain residence or office location and, thereby, used to determine the user's identity.

Several other types of private information such as health issues or religious preferences surprisingly can also be revealed by just observing the anonymous users' movements and usage patterns over time. In general, there is a close correlation between the identities of people and their movement patterns. Note that hiding the locations of users is much more challenging than hiding their identities. This is because, with location-based services, the location of the user is needed for successful data access or data collection but the identity of the user is not necessary.

There are many additional challenging research problems. For example, we do not know yet how to share private data while limiting disclosure and ensuring sufficient data utility in the shared data. The existing paradigm of differential privacy is a very important step in the right direction but it unfortunately reduces information content too much in order to be useful in most practical cases. In addition, real data is not static but gets larger and changes over time. None of the prevailing techniques results in any useful content being released in this scenario. Yet another very important direction is to rethink security for information sharing in cases where Big Data is used. Many online services today require us to share private information. However, beyond record-level access control, we do not understand what it means to share data, how the shared data can be linked, and how to give users fine-grained control over this sharing.

Data ownership refers to both the possession of, and responsibility for, information. Ownership implies power as well as control. The control of information includes not just the ability to access, create, modify, package, derive benefit from, sell or remove data, but also the right to assign these access privileges to others [20]. Implicit in having control over access to data is the ability to share data. There are situations where sharing of data may not be appropriate. The key is to know the various issues impacting ownership and sharing of data and to make decisions that protect the interests of the parties involved [31]. As a by-product of information processing, data has both intrinsic value and added value. The degree of ownership and responsibility is a function of the value each interested party derives from the use of that information [20]. The cost and benefits of data sharing should be viewed from ethical, institutional, legal, and professional perspectives.

\section{Building business models in global data-driven ecosystems}

Sometimes it is difficult to understand where small business owners fit into the larger entrepreneurial sphere in which they operate. If, as the Internet of Things implies, all the technology in the world will eventually be connected to the Internet for easy control and access, what will be the impact on small businesses on both a micro and a macro level? Several important factors should be considered: 
- Progress will be gradual. Because the IoT will develop over time, small business owners should not fear that their company will immediately become obsolete. Perhaps some small business owners have already invested in technologies including wireless Internet, mobile devices, analytical software, cloud storage or virtual servers. Whether owners realize it or not, they have already begun to adapt to changes in the world around them. That progressive outlook has permitted them to stay on pace with the development of the IoT.

- Technological advances are already available and affordable. Some of the semiconductor components that are so central to most IoT applications have shown much more functionality at lower prices than were available in the past. Newer processes, such as the ARM Cortex M, use only about one-tenth of the power that most energy-efficient 16-bit processors used just two years ago. This leap forward in technological capabilities has been most apparent in the evolution of smart watches. While the first of these products released just four years ago boasted 400-megahertz single processors and simple three-axis accelerometers, today's smart watches include 1-gigahertz dual-core processors and high-end, six axis devices that combine gyroscopes and accelerators. Meanwhile, the prices of the chip sets used in these products have declined by about 25 percent over the past two years.

- Increased integration is useful. The emergence of more integrated system-in-package and system-on-chip devices is helping to overcome some of the challenges by addressing power, cost, and size factors.

- Emerging standards are promising. Over the past two years, semiconductor players have worked collaboratively with industry associations, academic consortiums as well as hardware, networking, and software companies to develop formal and informal IoT standards. AT\&T, Cisco, GE, IBM, and Intel have cofounded the Industrial Internet Consortium. Their primary goal is to establish interoperability standards across industrial environments. This would enable, for example, data about fleets, machines and facilities to be accessed and shared more reliably. Other groups have been focused on standardizing the application programming interfaces (APIs) that enable basic commands and data transfer among IoT devices.

- Connectivity standards are becoming superior. The current cellular, Wi-Fi, Bluetooth and ZigBee specifications and standards are sufficient to handle most IoT applications on the market. Some applications, however, require low-power, low-datarate connectivity across a range of more than 20 meters - an area in which cellular technologies and Wi-Fi often fall short. New technologies to address this need are emerging from sources including Bluetooth and
Weightless interest groups. The Weightless Special Interest Group (SIG), for example, is comprised of technology companies exploring the use of free wireless spectrum to establish an open communications protocol. These standardization efforts will enable those IoT applications that require broadly distributed sensors operating at lower power over a low-cost spectrum. Those applications might include temperature and moisture sensors used in agricultural settings.

- Sensor technology will increasingly drive the IoT. Devices can be programmed to sense the environment and to share that information, such as the location of items or temperature readings, over the Internet. Small business owners who understand how, when, and where sensors can be used will benefit the most from new and existing technology.

- Small businesses can become more efficient. The IoT, together with Big Data, will enable small businesses to operate more efficiently and to use their resources more effectively. Information will be provided on energy use and associated technologies such as thermostats, air quality monitors, lighting, and building security. For companies in service industries, remotely connected devices and sensors may allow service technicians to assess and monitor issues without having to make time-consuming trips.

- Security will become more important. More data, information and connectivity come with a need for better security. Small companies must supplement new technologies with additional security measures and preventive action.

- Data and the IoT have been backed by influential supporters. These concepts are not just some new ideas with little traction. Rather, they are serious issues with worldwide powerful and influential supporters.

- The attention of suppliers has increased. IoT developer tools and products are now available. Apple, for instance, has released HealthKit and HomeKit developer tools as part of its latest operating system upgrade. Google has acquired Nest to catalyze the development of an IoT platform and applications.

- The impact of consumers on the IoT is significant. Demand for the first generation of IoT products (e.g., fitness bands, smart watches, smart thermostats) has increased as component technologies have evolved and their costs have declined. A similar dynamic occurred with the rise of smartphone usage. The increase in orders coincided with a steep decline in the price of critical smartphone components.

- Business opportunities will increase. Numerous industry experts predict that hundreds, if not thousands, of unique business opportunities will arise from the growth of the IoT. Specific areas include increased customer service and personalization; the ability to 
address real-time threats, demands, and needs; improved process optimization; and more accurate and effective forecasting.

The most popular IoT applications and devices include:

- Smart Home. This is likely the most popular IoT application at the moment because it is most affordable and readily available to consumers. The Amazon Echo and Google Nest are among the hundreds of products on the market that users can control by using voice commands to make their lives more connected than ever. Users of Amazon Echo, for example, can tell its voice assistant, Alexa, to perform a variety of functions such as playing music, providing weather reports and sports scores, or even ordering an Uber.

- Smart Wearables. Watches and wearables are no longer just for telling time. The Apple Watch and other smartwatches on the market have turned into smartphone holsters by enabling text messages, phone calls, and more. Devices such as Fitbit and Jawbone have helped to revolutionize the fitness world by giving people more data about their workouts. The Fitbit One, for example, tracks steps, floors climbed, calories burned and sleep quality. The device wirelessly syncs with computers and smartphones to transmit fitness data in a chart format to monitor an individual's progress.

- Smart Cities. The IoT has the potential to transform entire cities into smart cities by solving real problems faced by citizens daily. With the proper connections and data, the IoT can solve traffic congestion issues and reduce noise, crime and pollution. Barcelona, Spain, one of the foremost smart cities in the world has initiated several IoT initiatives to enhance smart parking and the environment.

- Smart Cars. Connected cars and vehicles are increasingly equipped with Internet access and can share that access with others, just like connecting to a wireless network in a home or office. AT\&T, for example, added 1.3 million cars to its network during the second quarter of 2016, bringing the total number of cars it connects to 9.5 million. Drivers don't have to subscribe or pay a monthly fee for data to participate in the AT\&T program.

Unlike managers at large corporations, small business owners and entrepreneurs have more freedom to explore and experiment with new ideas. We propose several opportunities to be explored:

- Medicine. Hospitals collect a massive amount of data. With the IoT, this data could be channeled into activities to dramatically improve care, reduce the length of stays and lower the transmission of diseases and infection rates.

- Manufacturing. The typical manufacturing facility might be made up of hundreds of tools and processes producing intelligent data that could be used to improve efficiency. The IoT could deliver this information to a central intelligence forum, where it could then be used to more quickly improve processes and achieve operational goals.

- Retail. Historically, retail operations have had very few functions fully connected. The IoT can play a central role in delivering the world-class consumer experience demanded by customers and allow for innovation, an increase in sales and a loyal customer base.

Small business owners and entrepreneurs will have opportunities created by investment and supported by data. Delivering data accurately will provide the greatest prospects. Key products and services that might be considered by enterprising parties include:

- Sensor enablement. The development of sensors for the machines, gadgets, transportation systems and people that fuel IoT will continue to grow.

- Data analytics. The need is increasing for intelligent software that can perform this type of analysis for business-minded end users for decision-making.

- Infrastructure investment. The opportunities to help deliver and consult related to the network infrastructure expansion continue to expand.

The Internet of Things is slated to change business as usual. It will provide the opportunity to create unique, practical and innovative products and services for a new generation of consumers. Getting there will require hard work and ingenuity. Small business owners and entrepreneurs are suited to deliver just that.

However, to succeed, small companies and start-ups will ultimately have to make a very crucial decision concerning their business models: either emulate Apple or emulate Micron. Therefore, they need to either focus on building a somewhat vertically integrated "product" company that sells a complete service under its own brand to consumers and businesses, or focus on being an embedded background player providing services or technology for established brands.

\section{Data and IoT service-based business models}

The rapid pace of technological change has been cited most often as the single biggest challenge facing companies [24]. The Internet of Things will alter industry structure and the nature of competition as it reshapes industry boundaries and creates entirely new industries [26].

Companies will be compelled to consider what type of businesses they are [26]. In a recent survey of Fortune $500 \mathrm{CEOs,} 67 \%$ indicated that, regardless of 
product or industry, they now consider their companies to be technology companies [24].

It is anticipated that the commercial exploitation of this technology will result in the development of new applications and the wider deployment of existing applications [10]. The IoT will be a major element for aligning technology development and economic value creation [8]. External factors, like technological innovation, will stimulate the development of new business models [5, 15].

With the pace and scope of change related to the Internet of Things, managers are baffled by their choices for business models. The traditional models, often based on strategic management theories, may no longer exclusively apply to this changing landscape. To stay competitive, companies will need to change their model holistically, adapting and innovating in every dimension $[5,33]$.

A business model describes the rationale of how an organization creates, delivers and captures value [25]. A review of the literature reveals that business frameworks exist to facilitate the development of new business models. The most normally used framework, the Business Model Canvas [25], identifies building blocks or components including customer segments, value propositions, channels, customer relationships, revenue streams, key resources, key activities, key partnerships, and cost structure $[8,25]$.

Several IoT models have been developed $[5,8,17$, 19, 32]. The model specifically for IoT applications developed by Dijkman, Sprenkels, Peeters, and Janssen (2015) [9], was derived from literature and with practitioners in the IoT domain. Their research demonstrated that the most significant building blocks for IoT business models were value proposition, customer relationships, and key partnerships [9]. A combination of these components is most important [9]. The value proposition addresses what is delivered to the customer. It creates value for a customer segment beyond the product or service offered and describes the value in quantitative (i.e., price or speed of service) or qualitative (i.e., brand, design, customer experience, cost/risk reduction) terms [2, 4, 25]. Within the value proposition building block, convenience, performance and cost reduction could be considered the most important types [8].

The customer perspective includes the customer segments served by the company, the channels used to reach customers, and customer relationships [5]. The model for IoT applications will most likely focus on co-creation and communities [9]. Key partnerships with software and app developers, launching customers, hardware partners and data analysis partners are critical to shaping the IoT business models [10]. Quinn (2000) has observed that innovations combining software and technology are prone to be outsourced [28]. Dijkman et al. (2015) have suggested that incorporating IoT products into the product portfolio may be a specialization that is partly acquired through outsourcing [10].

The Internet of Things is changing how value is created for customers and how competition among companies and industries is defined [26]. There are implications for business model innovation organizations of all sizes and types, in both the public and private sector. If, as the Fortune 500 survey indicated, every company is a technology company [24], new business models will be needed to leverage the power of the IoT.

Entrepreneurs will be needed who can create new industries and new wealth from technological innovations [4]. The barriers to entry for smaller and more innovative competitors will be lower as a result of the developments in technologies [4]. Improvements in market and customer intelligence in every sector, gained by unlocking the insights available from Big Data, will support entrepreneurial activity [7]. New business start-ups will be attracted by the possibility of strategizing more precisely while reducing the level of uncertainty for their decision-making [7]. Smaller businesses will be able to compete more effectively with larger, more-established firms because of the enhanced information and their ability to react more quickly and efficiently to changes in the market [7]. To prepare for these changes, managers should begin to collect more data, recruit and retain the best digital talent, retrain existing staff and plan how to restructure the organization [4].

Competitive advantage can be gained by scanning the environment for emerging opportunities created by the Internet of Things. Big Data is significant in its ability to provide information and knowledge for decision-making [10]. The historic and real-time data generated through supply chains, production processes and customer behavior provide a host of opportunities to generate wealth when properly analyzed to reveal valuable insights [9]. The main categories of organizations that can benefit from Big Data analytics include manufacturing, retail, central government, healthcare, telecommunications, and banking $[7,9]$.

Chan (2015) discussed how organizations have already begun to integrate Big Data and IoT applications into their business models [7]. For example, Smart TVs installed at shopping malls provide an opportunity for companies to evaluate the effectiveness and return on investment of digital signage and point-of-purchase displays through Cloud Audience Analytics. A video "presence detection" solution, it provides shopper analytics with profiles by gender and age, for example. In real-time, appropriate 
advertising will be displayed and insightful information will be collected and reported to improve advertising effectiveness.

Collaborators include the provider of the analytic software and gateway; the provider of the mobile network and Cloud facility; and the advertiser that provides the advertising message. Each of the collaborators benefits from this arrangement. The advertiser receives data concerning the viewer profile, the return on investment and the effectiveness of the advertisement. The provider of the digital signage with the camera and transmission modem receives revenue from its role as the cloud service platform. Finally, the provider of the data analytics is enriched by the imposition of service charges.

Other companies recognize their role as providers of after-sale services, remotely monitoring and diagnosing issues in the system. Companies can apply technologies such as RFID to their specific industry and collaborate with other parties in their ecosystem [7].

Information platforms offering comprehensive public transportation information can be developed using open data released by the government together with data collected from the Internet to offer comprehensive and up-to-date information for users. A large customer base can be built for use with advertising and other add-on features. Using a digital freemium business model, the application can be offered at no cost for users with its main source of revenue from mobile advertisements. Collaborators include complementary service providers, such as food delivery and taxis, which rely on location-based technology and generate revenue through service charge [7].

The use of the components of value proposition, customer relationships, and key partnerships identified by Dijkman et al. (2015) will increase in importance as companies seek to develop their business models in response to the evolution of the Internet of Things [9].

\section{Analysis of business opportunities in data analysis}

Whereas we do not discuss business models for the IoT equipment and infrastructure here, we have to underline that the provision of services based on IoT is dependent on the existence and access to the IoT infrastructure. Therefore, the speed with which the service market can develop may depend on the roll-out of suitable IoT infrastructure.

It may be expected that organizations will invest in data collection with immediate commercial (direct or indirect) interest and will be hesitant to invest in data collection that does not immediately add to productivity or quality. For Big Data applications, organizations may therefore have difficulty getting access to the necessary data sets.

Suitable data sets may exist within the primary interest of other organizations, therefore also subject to considerable privacy and confidentiality considerations and possibly financial obligations and restrictions.

One solution for this issue could be industry-wide access agreements across the sectors for this purpose. Another solution, similar to one used by media services, could be the provision of access to data sets or packages of data sets via a broker or packager, possibly adding another level of anonymity. However, that, by itself, may also compromise the usefulness for Big Data purposes as well as for regulated clinical medical tests.

The expansion of the Internet of Things (IoT) and Big Data will have significant implications for small businesses. Currently many enterprises are not able to take advantage of the IoT and Big Data. At present, only the largest of enterprises have access to the level of required resources. This means, then, that the large companies have often dominated sectors of industries as they relied on their extensive resources (including financial and informational) to perform analysis and to support the required infrastructure. Larger operations, such as Coca-Cola, General Electric and Domino's Pizza, have already begun to use the resources of Big Data and the IoT to provide value to their organizations [13]. Tata Consultancy Services recently reported that 26 companies, 14 of them in the United States, plan to spend $\$ 1$ billion or more each on IoT initiatives [12, 29]. The requirement of heavy capital investment created significant barriers to entry and prevented a level playing field for small businesses [12]. The cost of IoT systems is expected to drop and the use of cloud services to store and analyze data will become more prevalent. These factors should reduce operating expenses and make the use of the Internet of Things and Big Data more affordable for small businesses. As digitalization becomes more commonplace, barriers to entry will be reduced. As a result of these technologies, products and services will be commoditized, with small businesses competing with large companies for individual modules. The "plug and play" nature of digital assets will cause the value chains to disaggregate. Existing value chains will change resulting in opportunities for new business models [16]. This will permit focused, fast-moving competitors, many of whom can scale up more rapidly and at a lower cost, to enter the market [15]. Entrepreneurs, using mined data to determine preferences of customers, will "cherry pick" subcategories of products and undercut prices on small volumes. This will force larger companies to do the same. Porter and Heppelmann (2014) predict that, as 
the use of the IoT becomes more prevalent, the bargaining power of buyers and sellers will increase and the level of competition will significantly increase [26].

The analytics tools required to process large volumes of data are becoming more accessible to small businesses. These organizations will benefit by adopting big data strategy [13]. New market entrants, because of their size, may not have sufficient human resources and thus may need to rely on experts to provide technical solutions. However, there is a danger implicit in this arrangement since managers of small businesses may not have the expertise to supervise the experts. By concentrating the responsibility for developing applications for the IoT and Big Data functions primarily with outside consultants, there is an increased risk of conflicts of interest and potential ethical violations.

In a survey of small businesses conducted by AVG in $2014,57 \%$ of the respondents expect the IoT to have a significant impact on their bottom line. The opportunities for the use of the IoT are limited only by the imagination of entrepreneurs [14]. Michael Feldman, a former executive with Bigbelly Solar, has identified the key elements for successful ventures: identify why a particular device should be connected, what data will be obtained, and what kinds of decisions can be made with that data.

Small businesses will have the opportunities to capitalize on the expansion of the Internet of Things in several ways:

- Improve the efficiency of internal operations;

- Create innovative products and services for consumers and other businesses; and

- Provide technical and consulting services to other businesses.

The IoT can help small businesses to improve the efficiency of their internal operations. It is capable of providing control, optimization, monitoring and autonomy in organizations. For example, small businesses can use security alarms and other sensorbased systems, such as IoT thermostats and air conditioners to control air conditioning and heating costs [29]. In addition to monitoring machinery, the IoT is also affecting the world of work. If a workplace can be connected to the Internet, it can be have an IoT system. Activities in a factory could be monitored by relaying data to the cloud and providing trends and conclusions to users. With better access to data and the resultant better decisions, plants could be run more efficiently and more safely [21].

Employees will also be affected by the IoT and Big Data. Sociometric badges provided to employees can be used to measure behavioral response data and are mapped to important metrics including sales, revenue and retention. While large clients such as Bank of America and Deloitte are using the Humanyze sensors, one can only anticipate that the concept will eventually trickle down to small businesses eager to capitalize on the return on investment. The concept of "digital Taylorism," [1] with its focus on quotas and efficiency, has the possibility to affect knowledge workers as well. These innovations in large companies, once refined, will become standard metrics in organizations of all sizes. By identifying existing problems or ideas, entrepreneurs can develop solutions using IoT and Big Data to create innovative products and services. Josh Udashkin founded Raden in response to frustration with luggage handling at airports [27]. His company's smart luggage has sensors in its handle to turn the bag into an electronic scale. It has sensors that are paired to a smartphone so, for example, when persons are waiting for bags at the airport, an alert will let them know the bag is within 200 feet of the phone [30].

Finally, small businesses can develop business opportunities by providing technical and consulting services to other businesses. The value of IoT goes beyond the data. Services are still needed to protect the data, perform analysis, and present the findings [34]. Small businesses can be an integral part of these activities.

\section{Some simplified SME case studies}

In the following section, we give a brief description of some experiences of European small and medium enterprises (SMEs) with data analysis services related to the IoT and Big Data. We follow a critical approach and present anonymous data and information so as not to interfere with the interests of the companies involved.

We consider the following specific aspects and use different scenarios to illustrate the issues involved in each particular case:

- Collection of information and privacy while safeguarding privacy of customers and third parties;

- IoT data collection and data analysis; and

- Data collection suitable for Big Data purposes.

Collection of information while safeguarding privacy of customers and third parties is a major issue especially in small organizations. It is difficult there to divide responsibilities and to avoid conflicts of interest. Questions arise such as who controls whom, who has access to which customer's information, and do customers and third parties support or endanger privacy?

- Case 6.A: A small law office in Belgium. Contacts with customers and third parties go through a single email address. For important written information, 
privacy for client and third parties is an option, not a guarantee.

- Case 6.B: A medium size doctors' group in Switzerland. Contacts among customers (e.g., patients) and users are through a web-based, individualized communication system, with controlled, role-based access implemented for the group. However, customers and users tend to bypass what they perceive is a difficult system and instead use emails to others in the group, thereby undermining privacy protection. Furthermore, given the absence of effective standardization of eHealth interchange systems, the guarantee of privacy is compromised in communication among clinics and hospitals.

- Case 6.C: A small real estate management agency in France. Contacts among customers and users are through a web-based, individualized communication system, with controlled, role-based access implemented for the group. Even an organization with a small number of employees who can access financial data and information of the customers and users must protect their privacy interests.

Collecting data and data analysis is a major challenge even for larger organizations.

- Case 6.D: An elevator maintenance company in Italy. Modern elevators have sensors to gather data, but most installations do not support remote access to the observations collected. As a consequence, data analysis is a low priority for this company. This does not support access to a preventative maintenance market. Data analysis is done on an ad hoc basis without the support of specialized tools.

- Case 6.E: A utility meter data collection company in Italy. Energy consumption data is collected for town heating systems. Most installations do not allow remote, or even non-contact, meter reading because of perceived cost and privacy protection reasons. However, the Italian electricity distribution network is relatively far more advanced in supporting both remote and contactless access. As a consequence, data analysis is a low priority for this company. This does not support access to an analytics services market.

- Case 6.F: A utility meter data collection company in Belgium. All utility meter readings are collected for different distributor clients (i.e., water, electricity, and gas). Currently various methods of data collection exist including customer- and user-supplied information and ad hoc meter reading by agents. A transition is under way to implement remote and non-contact reading of meter information. Data analysis is applied to verify all meter readings, identify a fair percentage of errors in documented information in reports as well as detect malfunctioning meters. The company also provides an analysis of consumption patterns to the distributors who relay the information to their customers and users.
Currently, few small companies have the motivation and resources to apply Big Data techniques. In many cases, they lack the competence to even evaluate the possible use of Big Data. The scenarios discussed above illustrate that a favorable environment for Big Data applications does not exist at this time.

The exception is Case 6.F. Initially the objective of the company was to verify customer- and user-supplied meter readings to save costs for the company and its distributor clients. The use of Big Data techniques provided an analysis of consumption trends integrating historic consumption patterns with some other data such as temperature and holidays. These results could be of interest not only to the distributor clients but also to their customers and users. The company ensures privacy protection for customers and users with a webbased personalized system. However, customers accept the risk that Big Data techniques may make privacysensitive information more visible to the staff of both the utility meter data collection company and its distributor clients.

\section{Conclusions}

The offering and the size of the market for third party data analysis may be expected to develop as follows:

- The rate of development of the data services market will be limited by the speed of the roll-out of the relevant IoT infrastructure.

- For Big Data applications, dependency includes access to additional data sets, in turn depending on roll-out, privacy and confidentiality concerns as well as additional conditions and constraints.

- Small companies will call on affordable and less sophisticated data analysis services by third parties.

- Large companies will call from time to time on the services of highly specialized data analysts, who maybe better referred to as difficult problem solvers.

\section{References}

[1] "A modern version of "scientific management" threatens to dehumanise the workplace", Economist, Sept. 12, 2015, http://www.economist.com.

[2] Barbato, M., Industry 4.0. Professional Manager, Autumn 2015, pp. 51-55.

[3]Big Data, Gartner online glossary, http://www.gartner.com/it-glossary/big-data.

[4] Bucherer, E., and Uckelmann, D., "Business models for the Internet of Things ", Architecting the internet of things, Uckelmann, D., M. Harrison, and F. Michahelles, (Eds.), Springer Berlin Heidelberg, 2011. 
[5] IBM, Global CEO Study - The Enterprise of the Future, 2008, http://www03.ibm.com.

[6] Centre for Economics and Business Research (CEBR), Data equity: Unlocking the value of big data, 2012, http://www.sas.com.

[7] Chan, H. C., "Internet of things business models", Journal of Service Science and Management, 8(4), 2015, p. 552.

[8] Chesbrough, H., and R. S. Rosenbloom, "The role of the business model in capturing value from innovation: evidence from Xerox Corporation's technology spin-off companies", Industrial and corporate change, 11(3), 2002, pp. 529-555.

[9] Dijkman, R. M., B. Sprenkels, Peeters, T., and A. Janssen, "Business models for the internet of things", Int. Journal of Information Management, 35(6), 2015, pp. 672678 .

[10] Elgendy, N., and A. Elragal, "Big data analytics: a literature review paper", Advances in Data Mining. Applications and Theoretical Aspects, 2014, pp. 214-227.

[11] Fan, P. F., and G. Z. Zhou, "Analysis of the business model innovation of the technology of internet of things in postal logistics", Proceedings of industrial engineering and engineering management, 2011, pp. 532-536: IEEE Press.

[12] Foreman, M., "Internet of Things is web's next money spinner says small business", Nov. 18, 2014, http://now.avg.com.

[13] Hayles, W., "Why big data and the internet of things are a perfect match", Apr. 23, 2015, http://www.datamation.com.

[14] Hewlett Packard Enterprise (HPE), "Believe it or not, small business can use the internet of things", http://www.hpematter.com.

[15] Hirt, M., and P. Willmott, "Strategic principles for competing in the digital age", McKinsey Quarterly, 2014, http://www.mckinsey.com.

[16] Kiel, D., C. Arnold, M. Collisi, and K.- I. Voigt, "The Impact of the Industrial Internet of Things on Established Business Models", Proceedings of the 25th International Association for Management of Technology (IAMOT) Conference, Orlando, FL, 2015.

[17] Li, H., and Z. Z. Xu, "Research on business model of Internet of Things based on MOP", Proceedings of the international Asia conference on industrial engineering and management innovation, Germany, 2013.

[18] Lindsay, G., "HR Meets Data: How Your Boss Will Monitor You To Create The Quantified Workplace", Sept. 21, 2015, http://www.fastcoexist.com.

[19] Liu, L., and W. Jia, "Business model for drug supply chain based on the internet of things", Proceedings of the international conference on network infrastructure and digital content, 2010, pp. 982-986: IEEE Press.

[20] Loshin, D., "Knowledge Integrity: Data Ownership", June 8, 2004, http://www.datawarehouse.com.

[21] Lumb, D., "How the internet of things is changing work", Nov. 13, 2015, http://www.fastcompany.com.

[22] Manyika, J., M. Chui, B. Brown, J. Bughin, R. Dobbs, C. Roxburgh, and A.H. Byers, "Big Data: The Next Frontier for Innovation, Competition, and Productivity", McKinsey Global Institute Reports, 2011, pp. 1-156.

[23] Mayer-Schonberger V, and K. Cukier, "Big data: a revolution that will transform how we live, work, and think", Boston: Houghton Mifflin Harcourt, 2013.

[24] Murray, A., "Two Tales from the Digital Frontier", Fortune, June 15, 2016, http://www.fortune.com.

[25] Osterwalder, A., and Y. Pigneur, Business model generation: a handbook for visionaries, game changers, and challengers, Hoboken, NJ: John Wiley \& Sons, 2010.

[26] Porter, M. E., and J. E. Heppelmann, "How smart, connected products are transforming competition", Harvard Business Review, 92(11), 2014, pp. 64-88.

[27] Price, E., "Why this smart luggage startup thinks it can roll to the top", Mar. 29, 2016, http://fortune.com.

[28] Quinn, J. B., "Outsourcing innovation: the new engine of growth", MIT Sloan Management Review, 41(4), 2000, pp. 13-28.

[29] Shein, E., "How the internet of things is helping small business", Dec. 14, 2015, http://www.forbes.com.

[30] Shu, L., "Raden wants to be the Apple of luggage with a pair of smart suitcases", Mar. 29, 2016, http://www.digitaltrends.com.

[31] Sieber, J. E., "Sharing scientific data I: new problems for IRBs”, IRB, 11(6), 1989, pp. 4-7.

[32] Sun, Y., H. Yan, C. Lu, R. Bie, and P. Thomas, "A holistic approach to visualizing business models for the internet of things", Communications in Mobile Computing, 1(1), 2012, pp. 1-7.

[33] Venkatraman, N., and J. C. Henderson, "Four vectors of business model innovation: Value capture in a network era", From Strategy to Execution, Pantaleo D., and N. Pal (Eds.), Springer Berlin Heidelberg, 2011.

[34] White, E., "Big data and the internet of things", July 30, 2015, http://news.syscon.com. 